\title{
An immunogenetic study of familial scleroderma
}

\author{
M D de Juan, J Belzunegui, I Belmonte, J Barado, M Figueroa, J Cancio, S Vidal, \\ E Cuadrado
}

\begin{abstract}
Objective-To study the role of the HLA system in the genetic susceptibility to familial systemic sclerosis (SSc).

Methods-HLA class I antigens were determined by classic serological methods and HLA-DRB, -DQA and -DQB genes were analysed by genetic typing in 36 individuals belonging to two families with several individuals affected by SSc.

Results-The results did not show any association of the inheritance to SSc with any particular HLA allele in these families but revealed a striking frequency of ANA autoantibodies in healthy spouses of the members of these families.

Conclusion-The otherwise infrequent familial incidence of SSc does not appear to be primarily linked to the HLA system in this study but it is suggested that other unknown exogenous environmental factors could be implicated in the development of the disease in families.
\end{abstract}

(Ann Rheum Dis 1994; 53: 614-617)

Although systemic sclerosis (SSc) is not considered to be a primarily genetic disorder, it is generally accepted that the disease does have a genetic component. There are several reports of familial occurrence ${ }^{1-4}$ and certain alleles of the HLA system have been associated with the disease. ${ }^{5}$ Moreover, several groups reported the appearance of autoantibodies and clinical features related to the disease among first degree relatives of SSc. ${ }^{6}$ Conversely, an increased incidence of ANA in spouses of affected individuals has been reported. ${ }^{78}$ Although this fact has not been confirmed by others, ${ }^{9}$ it suggests that an exogenous component could be related to this disease.

The association of HLA with SSc has only been studied in a few cases of familial SSc probably due to the extremely low incidence of familial occurrence. The aim of this study was to analyse the association of the HLA system with the inheritance of scleroderma or its clinical and serological features in two families with more than one affected member.

\section{Patients and methods}

CASE REPORTS

The pedigrees and HLA haplotypes of both families are shown in the figure. Both families are of Basque origin and live in the same area of the Basque country, in the north of Spain.
Family 1

Subject $1-2$, the mother, diagnosed with rheumatoid arthritis, died of an unknown cause.

Subject I-1, the father, was healthy and died of an unknown cause before the onset of the study.

Subject II-5, the scleroderma proband, female, 49 years old, has had Raynaud's phenomenon since she was 35 years old. She has non-erosive polyarthritis in the hands and feet, sclerodactyly and a slight muscle weakness with moderately increased CPK level. Her serum was positive for ANA (titre 1/160) and no ENA specificities could be determined.

Subject II-7, the other scleroderma patient, a 63 year old woman, has had Raynaud's phenomenon since she was forty, skin thickening and induration and sclerodactyly in the hands and arms. She also has telagienctasias and subcutaneous calcinosis. Pulmonary function tests showed a marked diffusion abnormality ( $50 \%$ of predicted). She had no radiological abnormalities and a barium oesophagram demonstrated hipomotility and dilatation in the distal oesophagus. She also presented with Sjögren's syndrome. Her serum was positive for ANA at titre $1 / 80$ and also for anticentromere antibodies, titre $1 / 80$. ENA was positive for SSb/SSa specificities and rheumatoid factor was positive (titre $1 / 2560$ ).

Subject II-1, a 58 year old man affected with Raynaud's phenomenon for years and positive ANA at titre $1 / 40$ with ENA negative.

Subject II-8, a 54 year old woman diagnosed with discoid lupus erythematosus. Her serum was negative both for ANA and ENA autoantibodies.

Subject II-6, a 61 year old woman, complained of frequent arthralgias and ANA positive at low titre: $1 / 40$.

Subject III-12, a daughter of the scleroderma proband presented ANA positive (titre 1/40) and ENA negative.

The spouses of subjects II- 4 , II- 5 , and II- 8 were found positive for ANA at titres ranging from $1 / 40$ to $1 / 80$ with speckled or nucleolar patterns.

\section{Family 2}

Subject II-2 The proband, a 56 year old woman, has had Raynaud's phenomenon since she was thirty six years old, sclerodactyly, telangiectasias, sporadic arthralgias and a slight decrease of $\mathrm{CO}_{2}$ pulmonary diffusion. Her serum was positive for ANA at high titre $(1 />1280)$ and it became positive for anticentromere antibodies (titre 1/1280) several years ago. No ENA specificity could be determined. 
Family 1

III

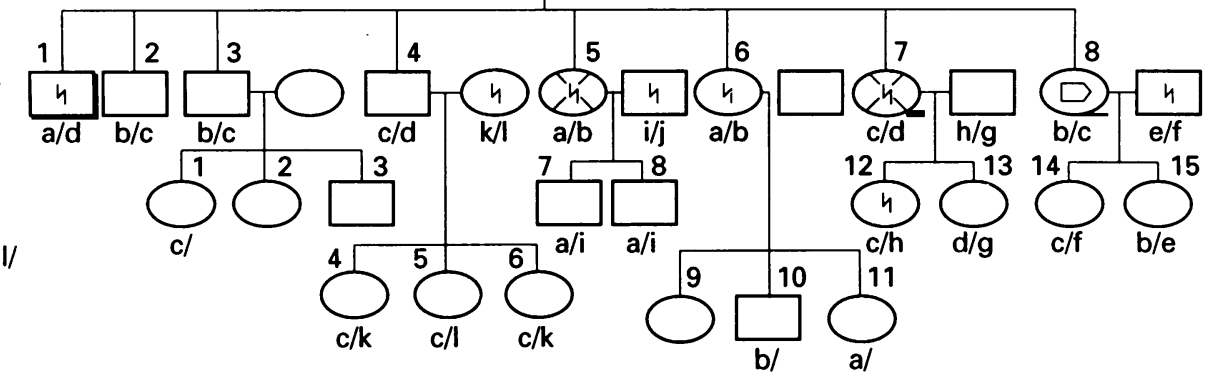

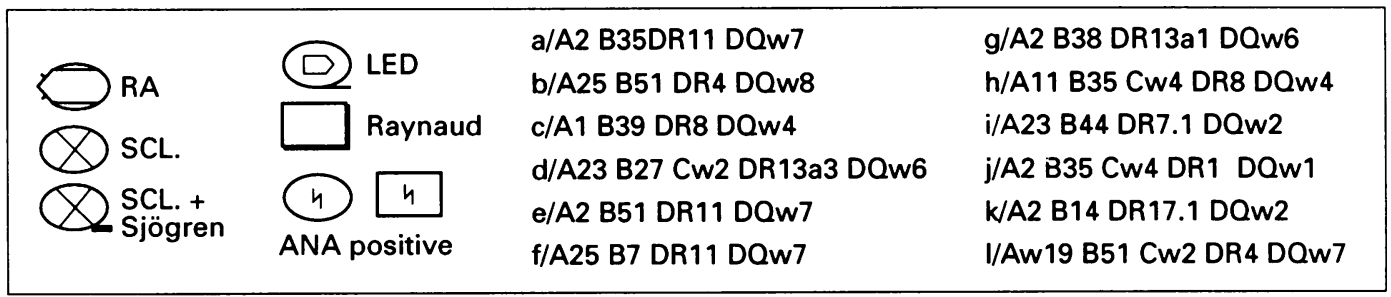

Family 2

l/
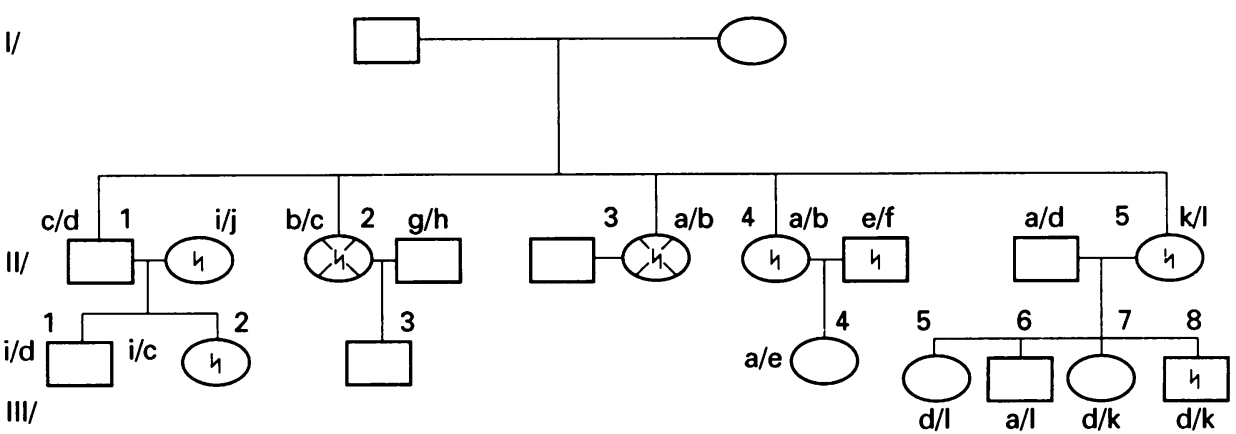

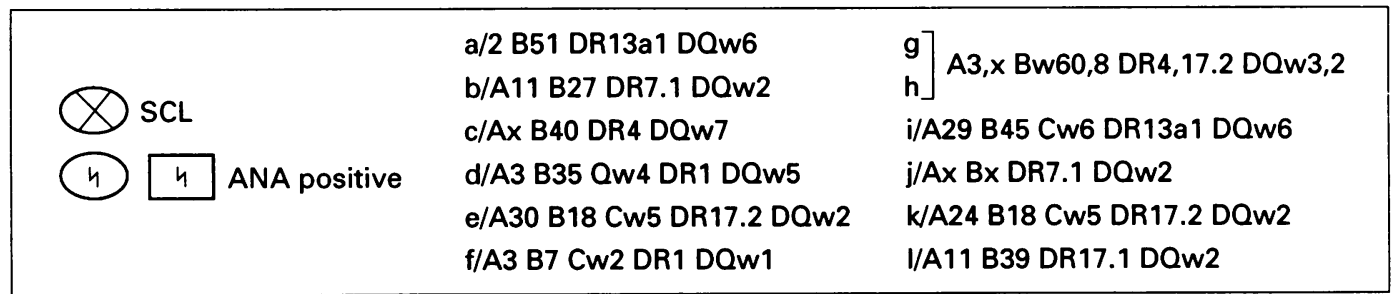

Pedigrees of families 1 and 2 with HLA haplotypes and serological features.

Subject II-3 The other affected subject, a 61 year old woman, has shown Raynaud's phenomenon since she was young. Previous records reported swollen hands but she is now free of sclerodermatosus skin changes. She suffered telangiectasias in the face, frequent arthralgias and a facial lupus-like rash for years. She has had ANA at variable titre and is negative for ENA both in CIE and immunoblot. Anti-centromere antibodies were positive on three occasions in the last two years.

Subject III-2 A healthy 16 year old woman, presented with a positive ANA serum at medium titre $(1 / 160)$ with a nucleolar pattern. Subjects II-4, II-5 and III-8 Healthy first degree relatives of the patients had positive
ANA tests at titres $1 / 40-1 / 80$, with speckled or nucleolar patterns. No ENA specifity was found among them. Three of the four spouses studied in the family presented ANA positive (1/40) sera.

\section{METHODS}

Complete medical studies and physical examinations were performed on all living family members. HLA class I (-A, -B, -C) typing was carried out using the microlymphocitotoxicity method $^{10}$ and HLA class II (-DR and -DQ) antigens were determined by RFLPs technique, as previously described. ${ }^{11}$ DRB, DQA and DQB specific probes were used to obtain the correspondent allogenotopes of 
HLA-class II alleles. Autoantibodies screening was carried out using Indirect Immunofluorescence (IIF) method, in rat liver, kidney and stomach sections. The Hep-2 epiteloid human cell line was also used to study ANA and $C$ Lucillae substrate was used to determine the presence of anti-double-stranded DNA antibodies. ENA specifities were studied by Ouchterlony immunodiffusion or contraimmunoelectrophoresis (CIE) ${ }^{12}$ using rabbit thymus (Pel-Freez Biol Roger, Arkansas) and human placenta extracts. We also used immunoblotting method as described elsewhere ${ }^{13}$ for further characterisation of ENA specifities. Rheumatoid factor was determined by a Latex particle method (Behring Institute, Marburg, Germany).

\section{Results}

HLA ANTIGENS AND AUTOIMMUNE DISEASE

The figure shows the HLA haplotypes segregation in both families. In family 1 , an important incidence of autoimmune disease and autoimmune features was found. Neither scleroderma patients nor the individuals affected with autoimmune diseases share any HLA particular haplotype. HLA B35 and DR1 1 or DR4 alleles, carried by the proband II-5, in haplotypes a and b, have been reported in association with scleroderma in other studies ${ }^{5}$ but in this case, these alleles are also found in both two healthy first degree relatives and they are absent in the other patient affected with scleroderma and Sjögren's syndrome. The analysis of family 2 also revealed a lack of association with HLA system and the inheritance of scleroderma. Here, both patients shared haplotype $\mathrm{b}$ (A11, B27, DR7) which is also carried by a healthy sister.

\section{AUTOANTOANTIBODY RESPONSE AND HLA SYSTEM}

There is no association between the appearance of autoantibodies (ANA) and any HLA antigen in patients and first degree relatives in either family. Anti-SSa/SSb and anticentromere responses followed the patterns of HLA association recently described by several studies $^{14}{ }^{15}$ but we did not find any scleroderma specific autoantibody (anti-topoisomerase I (Scl 70) or anti-centromere) in any first degree relative or other non-affected members of either family. Moreover, we observed a high prevalence of ANA autoantibodies (5/10, 50\%) in the spouses of the patients and other members of both families when compared with the ANA prevalence in their first degree relatives $(6 / 31,19 \%)$.

\section{Discussion}

In this study we report two new cases of familial scleroderma, a rarely reported condition. Several lines of evidence indicate a genetic predisposition to SSc. Autoantibodies characteristic of SSc are found in high frequency in blood relatives of patients with $\mathrm{SSc} ;{ }^{6}$ familial clustering of clinical features of SSc and related diseases have been reported ${ }^{3}$ and finally, many studies have found increased frequencies of MHC antigens associated with scleroderma. ${ }^{5}$ Recent studies have showed that the HLA class II loci, rather than class I alleles, are involved in SSc susceptibility. ${ }^{5}$ There are some reports of familial scleroderma, covering different populations and intrafamilial relationships. Most of them carried out HLA immunogenetic studies but there are few which included HLA class II genetic typing techniques; there is a great variability in the results. Whereas several reports show a direct correlation between HLA system and the inheritance of the disease, ${ }^{5}$ others have found a lack of association of any particular HLA antigen or haplotype with the appearance of scleroderma. ${ }^{2}$ Our results agree with those reports that found no segregation of MHC system with the inheritance of SSc when it appears in families. Neither a correlation between HLA system and autoimmune disease nor its clinical features could be demonstrated (family 1). This fact supports the view that HLA genes are not primarily implicated in the development of SSc in these families although a larger number of multicase families should be evaluated to determine the linkage between HLA and familial SSc. Other causes could explain the conflict between the reported studies: clinical heterogeneity of the disease has been associated with several HLA alleles depending on its clinical subsets ${ }^{5}$ and there is a high frequency of clinical overlapping of SSc with related connective tissue diseases, most of them with defined HLA associations, (for example, Sjögren's syndrome, rheumatoid arthritis, lupus erythematosus). Moreover, other unknown genes apart from HLA could be implicated in the development of the disease although a weak genetic component is expected to be demonstrated in SSc predisposition. On the other hand, a high prevalence of ANA is found among the spouses of the patients and of the other first degree relatives of both families studied: $5 / 10$ spouses $(50 \%)$, a higher ANA prevalence than the one found in our experience ( $5 \%$ ANA positives in 100 healthy blood donors studied, unpublished data) and that reported in controls by other studies. ${ }^{9}$ This finding might represent the existence of an environmental exposure shared by the members of these families and it supports the probable contribution of undetermined exogenous factors in the susceptibility to SSc.

1 Sasaki S, Yoshino H. Systemic sclerosis in mother and daughter. Arch Dermatol 1977; 113: 378-9.

2 Sheldon WB, Lurie DP, Maricq HR, et al. Three siblings with scleroderma (systemic sclerosis) and two with Raynaud's phenomenon from a single kindred. Arthritis Rheum 1981; 24: 668-76.

3 McGregor AR, Watson A, Yunis E, et al. Familial clustering of scleroderma spectrum disease. Am $\mathcal{F}$ Med 1988; 84: of scleroder.

4 Hietarinta M, Koskimies S, Lassila $\mathrm{O}$, et al. Familial Scleroderma: HLA antigens and autoantibodies. $\mathrm{Br} \mathcal{f}$ Rheum 1993; 32: 336-8.

5 Briggs D, Black C, Welsh K. Genetic factors in scleroderma Rheum Dis Clin North Am 1990; 16: 31-51.

6 Pereira S, Black C, Welsh K, et al. Autoantibodies and Immunogenetics in 30 patients with systemic sclerosis and their families. $\mathcal{F}$ Rheumatol $1987 ; 14: 760-5$. 
7 Maddison PJ, Skinner RP, Pereira RS, et al. Antinuclear antibodies in the relatives and spouses of patients with systemic sclerosis. Ann Rheum Dis 1986; 45: 793-9.

8 Whyte J, Stephens CO, Harvey GR, et al. Familial occurrence of antibodies to topoisomerase I. Arthritis occurrence of antibodies to top

9 Barnett AJ, McNeilage LJ. Antinuclear antibodies in patients with scleroderma (systemic sclerosis) and in their blood relatives and spouses. Ann Rheum Dis 1993; 52: 365-8.

10 Danilovs J, Terasaki PI, Park MS, Ayoub G. B-lymphocyte isolation by trombin-nylon wool. In: Terasaki PI, ed. Histocompatibility testing 1980. Los Angeles: UCLA Tissue Typing Laboratory, 1980:287-8.

11 Bidwell J, Jarrod E. HLA-DR allogenotyping using exonspecific cDNA probes and application of rapid minigel methods. Mol Immunol 1986; 23: 1111-6.
12 Greenwald CA, Peebles CL, Nakamura RM. Laboratory test for antinuclear antibody (ANA) in rheumatic diseases. Lab Med 1978; 19-27.

13 Juarez C, Vila L, Gelpi C, et al. Characterization of the antigen reactive with anti-Scl-70 antibodies and its application in an enzyme-linked immunosorbent assay. application in an enzyme-linked

14 Reveille JD, MacLeod M, Whittington K, Arnett FC. Specific amino acid residues in the secon hypervariable region of HLA-DQA1 and DQB1 chain genes promote the Ro (SSa)/La (SSb) autoantibody responses. F Immunol 1991; 146: 3871-6.

15 Reveille JD, Owerbach D, Goldstein R, et al. Association of polar amino acids at position 26 of the HLA-DQB1 first domain with the anticentromere autoantibody response in Systemic Sclerosis (scleroderma). f Clin Invest 1992; 89: 1208-13. 